\title{
Berechnung des Hochfrequenzverhaltens komplexer Strukturen mit der Methode gekoppelter Streuparameter - CSC
}

\author{
H.-W. Glock, K. Rothemund, and U. van Rienen \\ Institut für Allgemeine Elektrotechnik, Universität Rostock
}

Zusammenfassung. Es wird eine Methode zur Berechnung der Hochfrequenz-Eigenschaften komplexer Strukturen vorgestellt. Das Verfahren beruht auf der Zerlegung der Gesamtstruktur in einzelne einfachere Segmente, deren breitbandige S-Matrizen mit kommerziellen Programmen berechnet werden. Das Gesamtsystem kann von beliebiger Topologie sein, und die Zahl der die Segmente verkoppelnden HohlleiterModen ist nicht begrenzt. Als Ergebnis steht bei offenen Strukturen deren vollständige S-Matrix, bei abgeschlossenen deren Resonanzeigenschaften zur Verfügung. Die theoretischen Grundlagen werden beschrieben und die Anwendung mit Beispielen aus dem Gebiet der Teilchenbeschleuniger und zu Eigenschaften schwach elliptisch geformter Resonatoren illustriert.

A method called Coupled S-Parameter Calculation - CSC is described which is used to calculate the rf properties of complex structures, i.e. either their scattering (devices with ports) or their resonance properties. The method is based on the segmentation of the entire system into sections being less complex, the external calculation of the section's broadband S-matrices, and a combination scheme, which is applicable to any topology and number of modes. The method's principle is described. Examples from the field of particle accelerator cavities and about the properties of weakly elliptical resonators are given.

\section{Einführung}

Für die numerische Simulation von Streu- oder Resonanzeigenschaften eines Hochfrequenzbauteils muß normalerweise dessen Geometrie komplett auf einem Gitter diskretisiert werden. Bei komplizierten Anordnungen ergeben sich daraus schnell Problemgrößen von mehreren Millionen Gitterpunkten, die selbst auf Rechnern mit vielen hundert MByte Hauptspeicher und modernsten Prozessoren nicht oder nicht in ver-

Correspondence to: H.-W. Glock

(hans-walter.glock@etechnik.uni-rostock.de) tretbarer Zeit behandelbar sind. Daher ist es von großem Vorteil, wenn eine komplexe Struktur in mehrere einfachere Segmente zerlegt wird, die einzeln mit feldberechnenden Simulationswerkzeugen behandelt werden können. Dazu muß aber die Verkopplung zwischen den Feldern der Segmente korrekt beschrieben werden. Dies gelingt mit einer geringen Anzahl an Parametern, wenn die koppelnden Felder im Satz der Hohlleiter-Moden des Querschnitts an der Trennstelle zwischen zwei benachbarten Sektionen entwickelt werden. Jedes Segment ist dann durch seine - breitbandige - Streumatrix bezüglich dieser Hohlleitermoden zu beschreiben.

In Abschnitt 2 wird eine Methode zur Verkettung von Streumatrizen einzelner Segmente von Gesamtstrukturen beliebiger Topologie und bei beliebiger Anzahl zwischen den Segmenten koppelnder Moden vorgestellt. Dieses Verfahren bezeichnen wir in englischsprachigen Veröffentlichungen als Coupled S-parameter Calculation, kurz CSC. In Abschnitt 3 geht es um einige technische Aspekte des Gebrauchs von CSC. Anwendungsbeispiele sind im Abschnitt 4 erläutert.

\section{Theorie}

Gegeben sei ein (linerares, zeitinvariantes, jedoch nicht zwingend passives oder reziprokes) Hochfrequenzbauelement, das wie in Abb. 1 in mehrere Segmente zerlegt werden kann. Dabei wird vorausgesetzt, daß zur Entwicklung der die Segmente verkoppelnden Felder ein geeignetes Basissystem zur Verfügung steht. Die Verwendung der Hohlleitermoden des Querschnitts einer ebenen Trennung zwischen zwei benachbarten Segmenten als Basis führt in der Regel schon mit wenigen Moden zu einer angemessenen Feldapproximation. Diese Hohlleitermoden müssen für die betrachtete Frequenz nicht notwendig ausbreitungsfähig sein. Weiterhin wird vorausgesetzt, daß die Streumatrizen $\mathbf{S}_{j}$ aller Segmente bezüglich des gewählten Basissystems für jeden zu untersuchenden Frequenzpunkt bekannt sind: aus numerischer Simulation, analytischer Rechnung oder Messung. In einem ersten Schritt werden 


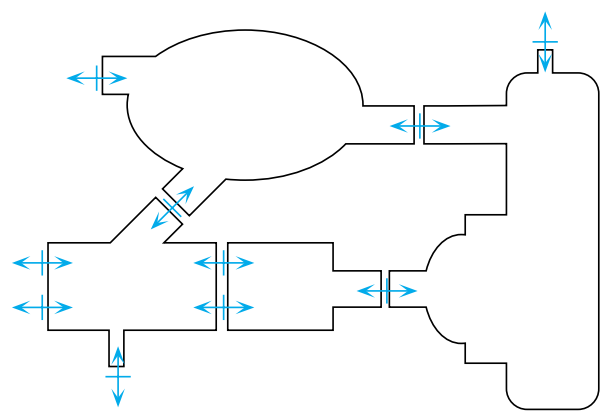

Abbildung 1. Beispiel einer zusammengesetzten Struktur mit internen Verzweigungen, Schleifen und Ports, an denen mehr als eine Mode verkoppeln.

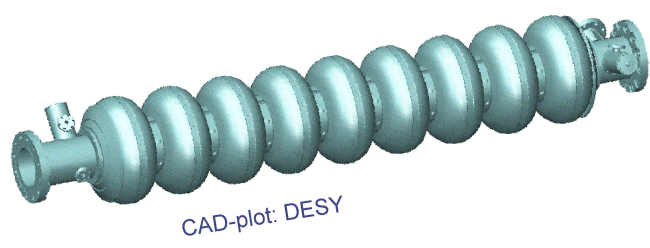

Abbildung 2. Supraleitend betriebene 9-zellige Resonatorstruktur zur Elektronen-/Positronenbeschleunigung. An beiden Seiten befinden sich Koppler.

diese zu einer Block-Diagonalmatrix $\mathbf{S}_{\text {all }}$ aller Streumatrizen zusammengefasst:

$\mathbf{S}_{\text {all }}(\omega)=\left(\begin{array}{ccc}\mathbf{S}_{1}(\omega) & 0 & 0 \\ 0 & \ddots & 0 \\ 0 & 0 & \mathbf{S}_{n}(\omega)\end{array}\right)$

Die Matrix $\mathbf{S}_{\text {all }}$ beschreibt zwar die Signalstreuung in allen einzelnen Segmenten, enthält aber keine Information über die Struktur des Gesamtsystems. Diese wird hinzugefügt, indem zunächst durch Anwendung einer Permutationsmatrix $\mathbf{P}$ die Reihenfolge der Signale so verändert wird, daß die innnerhalb des Gesamtsystems ausgetauschten Signale $\boldsymbol{a}_{c o p}$, die von außen einfallenden $\boldsymbol{a}_{i n c}$ sowie die nach außen gestreuten $\boldsymbol{a}_{s c t}$ blockweise zusammengefaßt sind. Eine weitere Permutationsmatrix $\mathbf{F}$ beschreibt die Nachbarschaftsrelationen der Segmente innerhalb des Gesamtsystems, also den Umstand, daß auslaufende Signale eines Segments die einlaufenden Signale des Nachbarsegments sind. In einfachen Systemen können $\mathbf{P}$ und $\mathbf{F}$ direkt angegeben werden; bei komplizierteren Anordnungen bedarf es einer automatischen Erzeugung. Insgesamt ergibt sich dann:

$$
\begin{aligned}
\left(\begin{array}{c}
\boldsymbol{a}_{c o p} \\
\boldsymbol{a}_{\text {sct }}
\end{array}\right) & =\mathbf{P}^{-1} \mathbf{F S}_{\text {all }}(\omega) \mathbf{P}\left(\begin{array}{l}
\boldsymbol{a}_{c o p} \\
\boldsymbol{a}_{\text {inc }}
\end{array}\right) \\
& =\left(\begin{array}{ll}
\mathbf{G}_{11} & \mathbf{G}_{12} \\
\mathbf{G}_{21} & \mathbf{G}_{22}
\end{array}\right)\left(\begin{array}{l}
\boldsymbol{a}_{c o p} \\
\boldsymbol{a}_{\text {inc }}
\end{array}\right) .
\end{aligned}
$$

Hieraus lassen sich mit den zur Abkürzung eingeführten Matrixblöcken $\mathbf{G}_{i j}$ die internen Signale $\boldsymbol{a}_{c o p}$ eliminieren:

$\boldsymbol{a}_{s c t}=\left[\mathbf{G}_{21}\left(\mathbf{1}-\mathbf{G}_{11}\right)^{-1} \mathbf{G}_{12}+\mathbf{G}_{22}\right] \boldsymbol{a}_{i n c}:=\mathbf{S} \boldsymbol{a}_{i n c}$

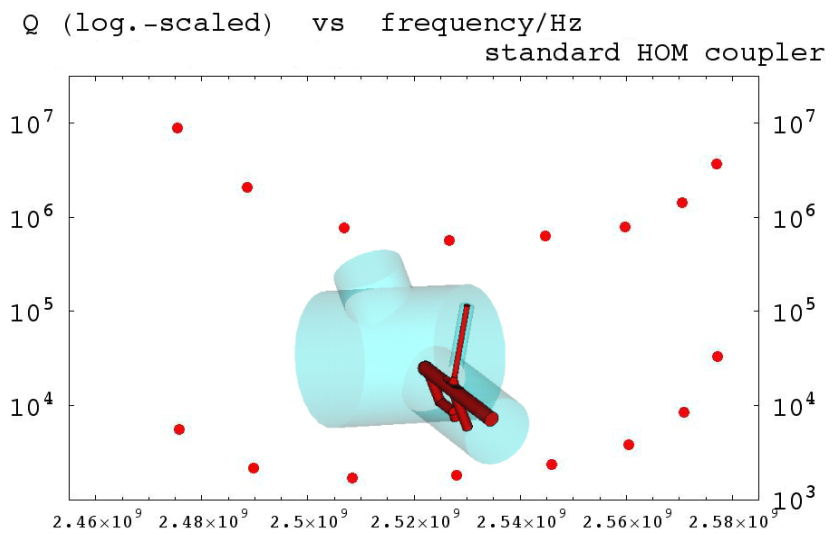

Q (log.-scaled) vs frequency/Hz

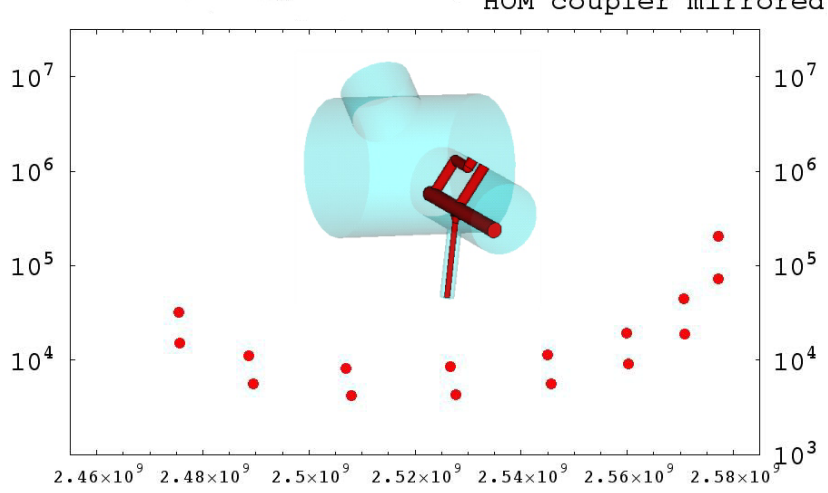

Abbildung 3. Güten, ermittelt aus Anpassung an Polsummen, für ursprüngliche Koppler-Anordnung (oben) und für gespiegelten und gedrehten linken HOM-Koppler.

Mit Gleichung (3) ist die gesuchte Streumatrix des Gesamtsystems explizit angegeben, sofern dieses mindestens einen offenen Port behält. Ist es hingegen vollständig abgeschlossen, kann eine nichttriviale Lösung nur für diskrete Resonanzfrequenzen gefunden werden, bei denen

$\left(\mathbf{1}-\mathbf{G}_{11}\left(\omega_{\text {res }}\right)\right) \boldsymbol{a}_{\text {cop }}=0$

gilt. Dies bedeutet, daß die Matrix $\left(\mathbf{1}-\mathbf{G}_{11}\left(\omega_{\text {res }}\right)\right)$ den Eigenwert 0 besitzen muß. In diesem (Resonanz-) Fall ist $\boldsymbol{a}_{c o p}$ der zum Eigenwert 0 gehörende Eigenvektor, der die Wellenamplituden der resonanten Feldverteilung darstellt. Numerisch findet man Resonanzfrequenzen durch wiederholte Suche nach dem Eigenwert 0 an allen Frequenzpunkten, an denen $\left(\mathbf{1}-\mathbf{G}_{11}(\omega)\right)$ bekannt ist.

\section{Implementation}

Die oben beschriebene Theorie, also insbesondere auch die automatische Erzeugung der Permutationsmatrizen, die Berechnung der Gesamtstreumatrix einer offenen Struktur für einen beliebigen Satz von Frequenzpunkten und die $\mathrm{Su}-$ che nach Resonanzen abgeschlossener Strukturen wurden in einem Mathematica ${ }^{\mathrm{TM}}$-Anwendungspaket (Wolfram Inc., 


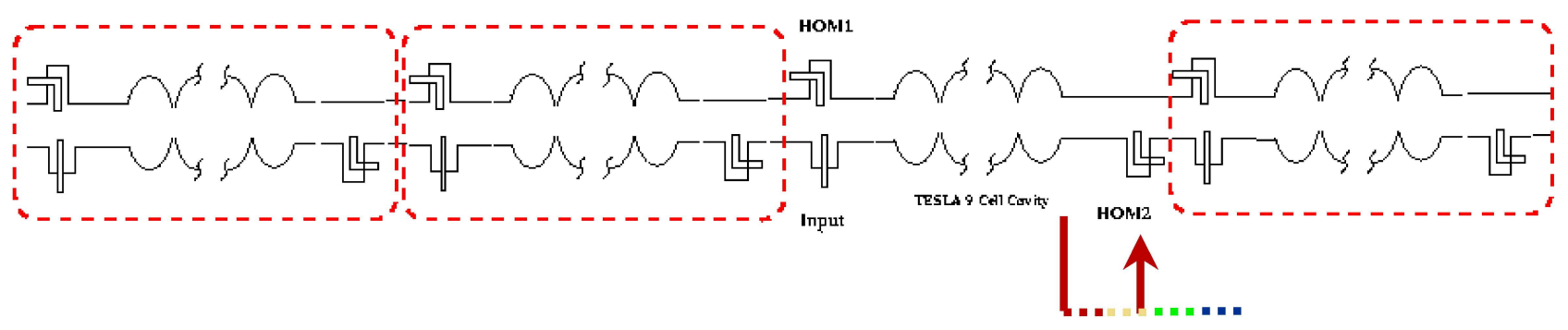

Abbildung 4. Kette aus vier Beschleunigungsresonatoren. Die Position des rechten HOM-Kopplers zu Resonator 3 wird variiert.

2000) implemetiert. Der Benutzer hat noch folgende Schritte auszuführen: 1.) Bereitstellung der Streumatrizen aller Segmente, tabelliert über die Frequenz. 2.) Erzeugung einer Liste der zur Gesamtstruktur gehörenden Segmente 3.) Erzeugung einer Liste der in der Gesamtstruktur gültigen Nachbarschaftsrelationen (jeweils in einer festgelegten Syntax). Alle weiteren Berechnungen werden von dem System erledigt. Zusätzlich zu den Basisfunktionen stellt die Implementation auch ergänzende Mechanismen wie die Interpolation von Streumatrizen auf Frequenzlisten, die allen Objekten gemeinsam sind, die analytische Berechnung der Streueigenschaften einfacher Objekte, sowie Datenein- und -ausgabenoptionen bereit. Die Verwendung des Pakets ist in Glock et al. (2001) ausführlich dokumentiert.

\section{Anwendungsbeispiele}

\subsection{Kavitäten zur Teilchenbeschleunigung}

In Abb. 2 ist ein Beschleunigungsresonator dargestellt, der in supraleitendem Zustand zur Elektronen-/Positronenbeschleunigung eingesetzt werden soll (DESY TDR, 2001). Der innere Teil besteht aus neun idealerweise rotationssymmetrischen Zellen. An beiden Seiten des Resonators befinden sich Koppler-Strukturen, die unerwünschte, vom Teilchenstrahl selbst erregte Felder in externe Dämpfungselemente ableiten sollen. Diese sog. HOM-Koppler haben eine komplizierte innere Form (DESY TDR, 2001), die eine sehr feine Diskretisierung bei der numerischen Berechnung (ca. 420 tsd Maschenpunkte pro Koppler) erfordern. Für eine angemessene vollständige Diskretisierung des gesamten Resonators einschließlich der Koppler würde man etwa 15 Millionen Maschenpunkte benötigen. Experimentell fand man bei der ursprünglichen Anordnung der Koppler unerwünscht stark ausgeprägtes resonantes Verhalten mit Güten zum Teil oberhalb $10^{6}$ (Baboi et al., 2000). In CSCRechnungen, bei denen die Gesamtstruktur aus den Elementen Strahlrohr - linker Koppler - Resonator - rechter Koppler - Strahlrohr zusammengesetzt wurde (Dohlus, priv. Komm., 2002), konnten diese Gütewerte bestätigt werden (vgl. Abb. 3, o.). Die Güten wurden dabei aus der Anpassung einer Summe von Polstellen an den von CSC berechneten breitbandigen S-Parameter-Verlauf ermittelt (Hecht et al., 2002). CSC-Vergleichsrechnungen, die von dem Vorschlag

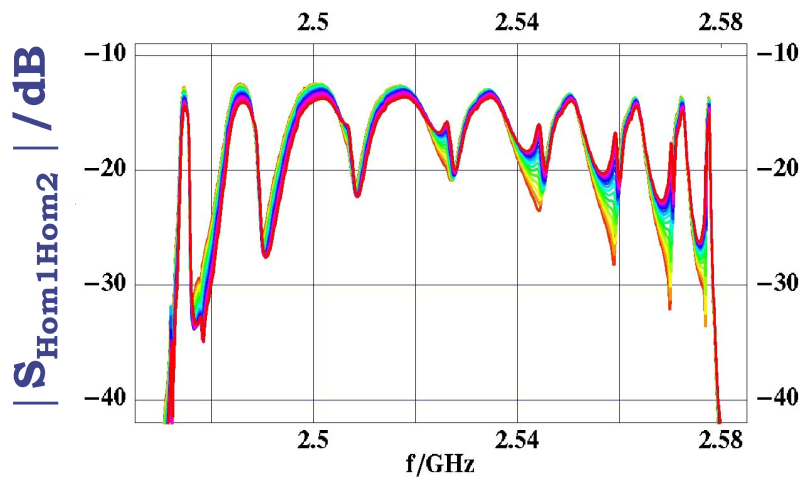

Abbildung 5. Transmission zwischen den Kopplern HOM1 und HOM2 einer Kette aus vier Resonatoren (vgl. Abb. 4) für verschiedene Positionen des Kopplers HOM2.

ausgingen, den linken der beiden Koppler verdreht und gespiegelt zu montieren (Dohlus, priv. Komm., 2002), zeigten, daß so eine sehr viel bessere Bedämpfung der unerwünschten Moden erreicht werden kann (Abb. 3, u.). Dazu mußte lediglich der modifizierte Koppler neu zur Berechnung seiner Streueigenschaften diskretisiert werden.

\subsection{Variation einer Kopplerposition}

Die zuvor beschriebenen Beschleunigungsresonatoren sollen zu längeren Ketten gruppiert eingesetzt werden. Es wurde untersucht, inwieweit die Verschiebung eines Kopplers entlang des Strahlrohrs zwischen zwei Resonatoren, wie sie aus Platzgründen wünschenswert schien, das Koppelverhalten beeinträchtigen kann. Es wurden zur Modellierung einer Kette von vier Resonatoren (vgl. Abb. 4) mit CSC die selben Grundelemente wie im vorhergehenden Beispiel verwendet. Allerdings wurde der Abstand des rechten Kopplers zu Resonator 3 in 31 Schritten variiert. Jeder dieser Schritte erforderte eine neue CSC-Berechnung, wobei die Streueigenschaften des runden Strahlrohrs für verschiedene Längen analytisch beschrieben werden konnten. Insgesamt benötigten diese Rechnungen 4300 s (Pentium III, $1 \mathrm{GHz}$ ), wobei intern Block-Streumatrizen der Dimension $84 \times 84$ zu Gesamtstreumatrizen der Dimension $16 \times 16$ an je 1000 Frequenzpunkten verarbeitet wurden. Eine vergleichbare direkte Berechnung in vollständiger Diskretisierung erscheint mit heute zur Verfügung stehender Computertechnologie ausgeschlossen. 


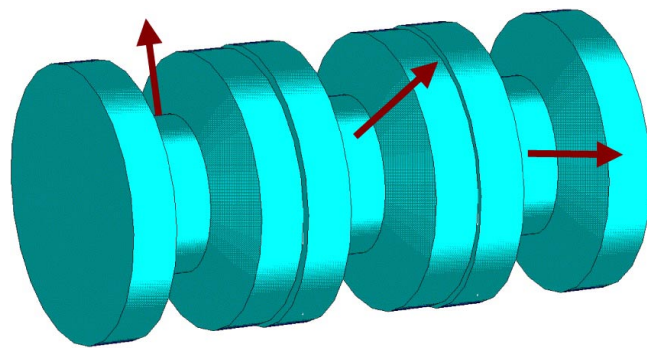

Abbildung 6. Kette aus drei Hantelzellen mit schwach elliptischen Querschnitten der Verengungen. Deren Orientierung ist jeweils gegeneinander um einen entlang der Kette konstanten Winkel $\varphi$ verdreht (angedeutet durch Pfeile).

Abbildung 5 zeigt als Resultat die vergleichsweise schwache Abhängigkeit der betrachteten Transmission als Kurvenschar.

\subsection{Dipol-Eigenmoden in schwach elliptischen Strukturen}

Im Gegensatz zu ideal rotationssymmetrischen Resonatoren, bei denen es keine ausgezeichnete Raumrichtung für die Orientierung polarisierbarer Moden gibt, kommt es bei noch so schwacher Abweichung von der Rotationssymmetrie - die technisch nie völlig vermieden werden kann - immer zu einer Festlegung der Feldorientierung. Damit einher geht eine Aufspaltung der Entartungspaare polarisierter Moden in zwei benachbarte Moden zwar sehr ähnlicher, aber nicht mehr identischer Feldverteilung, Energie und Resonanzfrequenz. Davon ausgehend wurde untersucht, wie sich Moden speziell in mehrzelligen Strukturen verhalten, in denen die ausgezeichnete Raumrichtung schraubenartig von Zelle $\mathrm{zu}$ Zelle um einen bestimmten Winkel verdreht wird. Als Modell diente eine Hantel-artige Zelle, deren mittlere Verengung schwach elliptisch (Halbachsenverhältnis 1.05) geformt waren (vgl. Abb. 6). Dazu wurde die Streumatrix einer einzelnen Zelle bezüglich der beiden $T E_{11}$-Polarisationen mit CST-MAFIA (2002) berechnet. Die Verkopplung mehrerer Zellen zu einem Resonator wurde mit CSC durchgeführt, wobei die Verdrehung von Zelle zu Zelle mit Hilfe virtueller Streuobjekte beschrieben wurde. Deren Streumatrix $\mathbf{S}_{\text {rot }}$ lautet für den vorliegenden Fall von zwei Dipol-Polarisationen für einen Drehwinkel $\varphi$ von Zelle zu Zelle:

$\mathbf{S}_{r o t}=\left(\begin{array}{cccc}0 & 0 & \cos \varphi & \sin \varphi \\ 0 & 0 & -\sin \varphi & \cos \varphi \\ \cos \varphi & -\sin \varphi & 0 & 0 \\ \sin \varphi & \cos \varphi & 0 & 0\end{array}\right)$.

Abbildung 7 zeigt einen Vergleich der so für ein Dipolmoden-Paar in Abhängigkeit von $\varphi$ ermittelten Resonanzfrequenzen mit den Resultaten direkter MAFIAEigenmode-Berechnungen. Letztere zeigen einen deutlich stärker streuenden Verlauf, was auf die winkelabhängige Qualität der Diskretisierung der elliptischen Bereiche auf dem kartesischen Gitter zurückgeführt wurde. Um dies zu überprüfen, wurden für einige Drehwinkel die direkte Be-

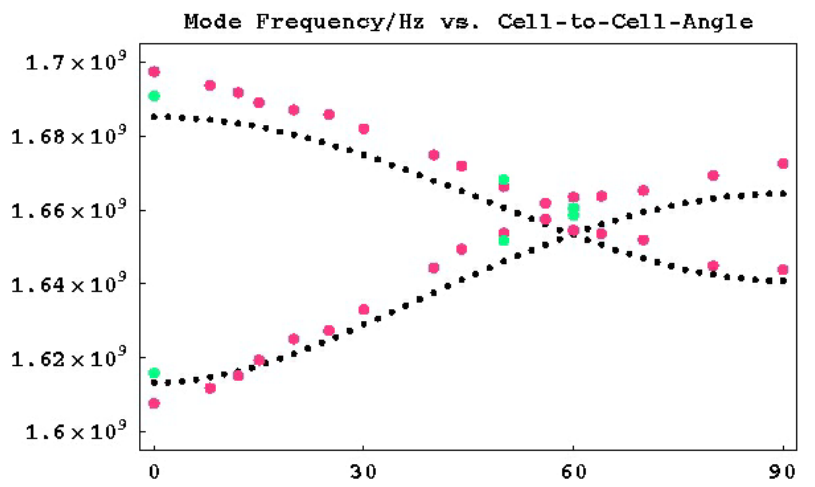

Abbildung 7. Resonanzfrequenzen eines ausgewählten Dipolmoden-Paares der Hantelzellenkette aus Abb. 6 in Abhängigkeit vom Drehwinkel Zelle-zu-Zelle $\varphi$ in Grad. Schwarze Punkte: CSCRechnung; rot (dunkel): direkte Rechnung mit MAFIA-E; grün (hell): MAFIA-E bei dreifacher Gitterpunktezahl.

rechnung mit einem Gitter dreifacher Punktezahl (1.85 Mio. anstatt 673 Tsd.) wiederholt. Dies führte zu einer deutlich besseren Übereinstimmung, insbesondere auch in der Umgebung des Entartungspunktes, der von CSC bei $\varphi=60^{\circ}$ gefunden wurde. Bemerkenswert sind die Unterschiede der erforderlichen Rechenleistung. Die CSC-Rechnung brauchte für jeden Winkel 40 s (PowerMac G3, $400 \mathrm{MHz}$ ), sowie einmalig ca. $3 \mathrm{~h}$ (SUN Ultra2, $350 \mathrm{MHz}$ ) zur Berechnung der S-Matrix einer Zelle. Die direkte Lösung mit MAFIA-E benötigte pro Winkel ca. $7 \mathrm{~h}$, auf dem verfeinerten Gitter ca. $22 \mathrm{~h}$ (jeweils SUN Ultra2, $350 \mathrm{MHz}$ ). In letzterem Fall wurde der von MAFIA adressierbare Speicher von 1.6 GByte RAM nahezu komplett ausgenutzt.

\section{Ergebnis}

Die Methode gekoppelter Streuparameter - CSC ermöglicht die Berechnung von Streu- bzw. Resonanzeigenschaften hochkomplexer Hochfrequenzstrukturen, basierend auf deren Zerlegung in einfachere Segmente mit einzeln zu berechnenden S-Matrizen. Das reduziert insbesondere dann den Gesamtaufwand, wenn Modifikationen einzelner Segmente interessieren oder analytisch beschreibbare Abschnitte (Hohlleiter, Rotationen) variiert werden. So können Anordnungen simuliert werden, deren direkte Berechnung Computer-Kapazität jenseits der heute verfügbaren Möglichkeiten erfordern würde.

\section{Literatur}

Baboi, N., Dohlus, M., Magne, C., et al.: Investigation of a High-Q-Dipole Mode at the TESLA Cavities, Proc. of the EPAC 2000, Vienna, http://accelconf.web.cern.ch/accelconf/e00/ PAPERS/WEP3B08.pdf, 1107-1109, 2000.

Brinkmann, R., Flöttmann, K., Rossbach, J., et al.: TESLA Technical Design Report, DESY 2001-011, DESY Hamburg, II-19 ff, 2001. 
CST GmbH, MAFIA Ver. 4, Büdinger Str. 2a, D-64289 Darmstadt. Dohlus, M.: DESY, private Kommunikation: S-Parameter des Resonators und Koppler-Geometrievorschlag, 2002.

Glock, H.-W., Rothemund, K., and van Rienen, U.: CSC - A System for Coupled S-Parameter Calculations, TESLA-Report, 2001-25, DESY Hamburg, 1-18, 2001.
Hecht, D., Rothemund, K., Glock, H.-W., et al.: Computation of RF-Properties of Long and Complex Structures, Proc. of the EPAC 2002, Paris, http://accelconf.web.cern.ch/accelconf/e02/ PAPERS/WEPRI060.pdf, 1685-1687, 2002.

Wolfram, S.: The Mathematica Book, 4th ed., Wolfram Media, 1999. 